\title{
o projeto 'Aulas em Casa' e a educação remota durante a pandemia do COVID-19: análise da experiência do estado do Amazonas
}

\section{The 'Aulas em Casa' project and remote education during the COVID- 19 pandemic: analysis of the experience of the state of Amazonas}

\section{Proyecto 'Aulas em Casa' y educación remota durante la pandemia COVID-19: análisis de la experiencia del estado de Amazonas}

Iolete Ribeiro da Silva ${ }^{1}$; Camila Ribeiro da Silva ${ }^{2}$

\section{RESUMO}

Em decorrência da pandemia do COVID-19 foram suspensas as aulas presenciais das escolas em todo o mundo. No Amazonas, as secretarias de educação do estado e do município de Manaus implantaram um projeto denominado 'Aula em Casa' que disponibiliza conteúdos através da televisão aberta, aplicativo de celular, YouTube, Facebook e um ambiente virtual de aprendizagem. $O$ objetivo deste trabalho foi analisar esse projeto a partir das narrativas de docentes sobre as condições de oferta do ensino remoto realizadas em Manaus/AM à luz da teoria histórico-cultural. Participaram do estudo seis docentes que atuam na rede pública estadual e municipal, que semanalmente produziram relatos de suas experiências durante o confinamento social. Foram identificados os principais obstáculos e analisadas as soluções adotadas pelo governo local. Conclui-se que não foram considerados os impactos da pandemia na vida das pessoas, os esforços centraram-se na transmissão de conteúdo desconsiderando a importância da mediação pedagógica. As saídas para uma questão tão complexa devem ser construídas em parceira com toda a comunidade escolar, em especial ampliando a compreensão do processo pedagógico.

Palavras-chave: Educação; Pandemia; Ensino Remoto; Amazonas.

\begin{abstract}
Due to COVID-19 pandemic, in-person classes all over the world were suspended. In Amazonas, the educational secretariats in the city of Manaus and state-wide, implemented a project named 'Aula em Casa' (as in Home Class) which enable content through open television, cellphone apps, YouTube, Facebook and a learning focused virtual environment. The objective was to analyse this project from the teacher's narratives regarding conditions and demand of remote learning in Manaus in the light of historic-cultural theory. Six municipal teachers participated of this study, which produced weekly reports of their experience during social confinement. The main obstacles and solutions proposed by the local government were identified. In conclusion, the pandemic impact on people's lives, the efforts to center the content transmission disregarding the importance of pedagogic mediation were all considered. The escapade for such complex question must be constructed in partnership with the whole educational community, especially amplifying the comprehension in the pedagogic process.
\end{abstract}

Keywords: Education; Pandemic; Remote Learning; Amazonas.

\footnotetext{
${ }^{1}$ Docente dos Programa de Pós-Graduação em Psicologia e em Educação da Universidade Federal do Amazonas (UFAM), Manaus/AM - Brasil. E-mail: ioleteribeiro@ufam.edu.br

2 Psicóloga e Pedagoga. Egressa da Universidade Federal do Amazonas, Faculdade de Educação. E-mail: camilaribeiro301@gmail.com
} 


\section{RESUMEN}

Debido a la pandemia de COVID-19, se suspendieron las clases presenciales en las escuelas de todo el mundo. En Amazonas, las secretarías de educación del estado y la del municipio de Manaus implementaron un proyecto denominado 'Aula em Casa' que ofrece contenidos por medio de la televisión abierta, una aplicación móvil, YouTube, Facebook y un ambiente virtual de aprendizaje. El objetivo de este trabajo ha sido el de analizar este proyecto a partir de las narrativas de los profesores acerca de las condiciones de la enseñanza a distancia que se ofrece en Manaus/AM a la luz de la teoría histórico-cultural. En el estudio participaron seis docentes que trabajan en escuelas públicas estatal y municipal, quienes semanalmente elaboraron informes de sus vivencias durante el aislamiento social. Fueron identificados los principales obstáculos, y analizadas las soluciones adoptadas por el gobierno local. Se concluye que no fueron considerados los impactos de la pandemia en la vida de las personas, y, que los esfuerzos se centraron en la transmisión de contenidos, desconociendo la importancia de la mediación pedagógica. Las soluciones a un problema tan complejo deben construirse en asociación con toda la comunidad escolar, en particular, ampliando la comprensión del proceso pedagógico.

Palabras clave: Educación; Pandemia; Enseñanza remota; Amazonas.

\section{INTRODUÇÃO}

A pandemia do COVID-19 afetou as escolas e suspendeu aulas presenciais em todo o mundo. Após o susto inicial os governos e sistemas de ensino começaram a discutir alternativas para retomada das aulas em segurança. No Estado do Amazonas, a opção da Secretaria Estadual de Educação, na capital, foi implantar um programa chamado Aulas em Casa. $O$ objetivo deste trabalho é analisar esse projeto a partir dos proposta pedagógica do Aulas em Casa e das narrativas de docentes sobre as condições de oferta do ensino remoto em Manaus/AM.

Este trabalho é parte de uma pesquisa maior sobre as trajetórias de escolarização de estudantes amazônidas, financiada pela CAPES e aprovada pelo Comitê de Ética em Pesquisa da Universidade Federal do Amazonas, CAEE 15366619.1.1001.5020, Parecer No 4.082.840. Foram analisadas a portaria, a resolução e as diretrizes pedagógicas do Projeto Aulas em Casa da SEDUC/AM, bem como os relatos de docentes que atuaram durante o primeiro semestre de 2020. Seis docentes da SEDUC participaram da pesquisa e registraram, uma vez por semana, durante 8 semanas, suas percepções sobre o trabalho no projeto Aulas em casa. Os registros, enviados para a primeira pesquisadora por meio de um aplicativo multiplataforma de mensagens instantâneas e chamadas de voz para smartphones, foram submetidos à análise interpretativa buscando-se identificar indicadores de sentido a partir do objetivo proposto na pesquisa. Os indicadores foram interpretados e reunidos em categorias.

No dia 13 de março de 2020 foi diagnosticado o primeiro caso de Covid-19 no Amazonas e, atualmente, o estado possui 185.932 casos confirmados e mais de cinco mil óbitos (FUNDAÇÃO DE VIGILÂNCIA SANITÁRIA, 2020). Manaus foi a primeira cidade do país a colapsar o sistema de saúde em razão do grande número de casos de pessoas infectadas pelo coronavírus simultaneamente. No dia 20 de abril, o sistema funerário iniciou a abertura de valas comuns nos cemitérios, pois, havia entrado em colapso em razão do grande número de mortos por COVID-19. No dia 24 de abril as Unidades de Terapia Intensiva (UTI) da capital estavam lotadas.

A pandemia seria por si só uma grave grive sanitária, mas no Brasil soma-se a ela a crise política e os efeitos de uma intensa desigualdade social. "E daí? Lamento! Quer que eu faça o quê?" foi o pronunciamento do atual presidente da república, enquanto o país atingia mais de 5 mil mortes por Covid-19. A pandemia aflorou a crise política já existente no Brasil, que somada ao negacionismo 
impactaram diretamente no comportamento da população durante essa crise sanitária. Outras desigualdades, dentre elas a educacional, se ampliaram demandando ainda mais o acesso às políticas públicas.

Mesmo antes da pandemia, os sistemas de ensino já enfrentavam problemas em relação ao acesso a vagas na educação infantil, acesso de jovens das camadas populares ao ensino médio e superior. A autonomia e liberdade de cátedra tem sido objeto de intensa disputa (MICHETTI, 2020), as propostas do programa escola sem partido, a militarização das escolas, os ataques às universidades e à área de Ciências Humanas e Sociais (GARCIA-PARPET, 2019). É nesse cenário de incertezas quanto ao desenrolar da pandemia e dos seus impactos sociais, intensificado diante da ausência de planejamento governamental para lidar com a crise, que analisamos o ensino remoto realizado em Manaus, uma das maiores cidade da Amazônia brasileira.

\section{O PROGRAMA AULAS EM CASA}

O regime especial de aulas não presenciais foi estabelecido no Amazonas em 18/03/2020 com a aprovação da Resolução No 30/2020 pelo Conselho Estadual de Educação do Amazonas (CEE/AM). Esta resolução definiu que as ações pedagógicas e administrativas deveriam ser planejadas em colaboração com o corpo docente (CONSELHO ESTADUAL DE EDUCAÇÃO DO AMAZONAS, 2020). Dois dias após a decisão do CEE/AM, a Secretaria de Estado da Educação e Desporto do Amazonas instituiu o regime especial de aulas não presenciais ao publicar a Portaria GS No 311/2020 (SECRETARIA DE EDUCAÇÃO E DESPORTO DO AMAZONAS, 2020a).

Para operacionalizar o regime especial de aulas não presenciais e o ano escolar de 2020, a SEDUC estabeleceu parceria entre o Centro de Mídias de Educação do Amazonas e a TV Encontro das Águas que mantem 3 canais de TV aberta para a transmissão de conteúdos educacionais voltados aos estudantes do $6^{\circ}$ ao $9^{\circ}$ ano do ensino fundamental e da $1^{\text {a }}$ a $3^{\mathrm{a}}$ Séries do Ensino Médio (SECRETARIA DE EDUCAÇÃO E DESPORTO DO AMAZONAS, 2020a).

A SEDUC (2020b) definiu que para os anos iniciais do ensino fundamental ( $1^{\circ}$ aos $3^{\circ}$ anos) seriam utilizadas atividades impressas e indicados portais de sites educacionais de acesso gratuito para ampliar o contato das/os estudantes com os conteúdos escolares. Nas diretrizes pedagógicas foram sugeridos aplicativos de jogos (Ler e contar, Todo Math, Piano Kids-music, Jogos educativos crianças 5, Game Kids, Mangahigh), programas de televisão (O shox da Luna, Dora, a aventureira, Peixonauta, Cocoricó, Castelo Rá-Tim-Bum e De onde vem?) e uma série de atividades para o desenvolvimento da coordenação motora, a aquisição do sistema de escrita e habilidades matemáticas. Tambem foram apresentadas sugestões para a Educação de Jovens e Adultos e para os $4^{\circ}$ e $5^{\circ}$ anos do ensino fundamental.

Para os anos finais do ensino fundamental ( $6^{\circ}$ aos $9^{\circ}$ anos) e para o ensino médio a SEDUC (2020b) definiu que estudantes deveriam acompanhar as aulas transmitidas pela TV Encontro das Águas (três horas de transmissão diária), acessar a conteúdos e recursos pedagógicos digitais disponíveis na plataforma Saber Mais e no ambiente virtual de aprendizagem. Outras estratégias complementares elencadas foram: sugestão de filmes, vídeos, documentários, sites, leituras e pesquisa e produção textual.

A operacionalização do regime especial de aulas não presenciais envolveu diferentes órgãos e departamentos tais como o Centro de Mídias de Educação do Amazonas (CEMEAM), a Assessoria de 
Comunicação da SEDUC/AM, o Departamento de Políticas e Programas Educacional, o Departamento de Gestão Escolar, o Centro de Formação Padre Anchieta, as Coordenarias Distritais e Regionais de Educação, a Assessoria de Acompanhamento e Avaliação das Políticas Educacionais e as Unidades Escolares (SEDUC, 2020b). A cada um desses órgãos/setores/departamentos foram estabelecidas responsabilidades relativas à produção de material, divulgação, definição de diretrizes pedagógicas. Destaca-se que a ação de formação de professoras/es referia-se somente à disponibilização de roteiros de estudo e divulgação de cursos on-line gratuitos e materiais de apoio.

Às Unidades Escolares cabia a execução das propostas deliberadas nos níveis centrais de gestão com pouco espaço para autonomia no planejamento pedagógico. As/Os docentes seriam os responsáveis por manter o contato com estudantes pais e responsáveis via aplicativos de mensagens instantâneas ou outros dispositivos de comunicação a distância para orientar sobre os estudos e realizar avaliações (SEDUC, 2020b).

Em 27 de março de 2020 a Secretaria Executiva Adjunta Pedagógica (SEDUC, 2020c) publica Nota anunciando a definição de diretrizes pedagógicas para o regime especial de aulas não presenciais por reconhecer que muitos/as estudantes não possuíam acesso a computadores e internet e seriam necessárias outras medidas para além das aulas televisivas. Esse documento reafirma que as ações educacionais desenvolvidas a distância serão contabilizadas como carga horária e dias letivos efetivados e que "não serão acolhidas iniciativas que forem tomadas de forma individual e que se distanciem do que esta legalmente proposto para o Regime Especial de Aulas Não Presenciais" (SEDUC, 2020c, p, 3).

Após reconhecer que muitos estudantes não conseguiam acessar as aulas por diversas razões: não possuíam internet, faltavam equipamentos, não havia um lugar adequado para estudar em casa, falta de conhecimento por parte de estudantes e familiares sobre as TIC ou mesmo porque residiam em localizadas que não possuíam cobertura da TV Encontro das Águas o que dificultava o acesso às plataformas educacionais a SEDUC começa a anunciar o retorno das aulas presenciais.

Manaus foi a primeira capital do país a anunciar o retorno das aulas presenciais tanto na rede privada quanto na rede pública. A reabertura das escolas privadas foi autorizada pelo Governo do Estado do Amazonas em 6 de julho de 2020 e o retorno das aulas do ensino médio da rede pública ocorreu em 10 de agosto de 2020 nas escolas da capital. Docentes passaram a ministrar, ao mesmo tempo, aulas pela internet e presenciais. As atividades presenciais passaram a ser escalonadas.

A decisão de retorno foi questionada por alguns pesquisadores em razão do risco de contágio, vez que a transmissão comunitária ainda estava vigente. Pesquisadores responsáveis pelo ATLAS dos Objetivos de Desenvolvimento Sustentável no Amazonas (2020), em 18 de agosto de 2020, recomendaram a suspensão das atividades presenciais nas redes pública estadual e privadas de ensino em Manaus. O ATLAS indicou a ocorrência de nova aceleração de casos e óbitos em agosto, com um aumento desproporcional no número de novos casos e óbitos entre pessoas jovens, entre 0 a 29 anos, especialmente entre as do sexo feminino desde a reabertura do comércio no dia $1^{0}$ de junho: "Nessas faixas etárias, o número de casos mais que dobrou apenas durante julho e isso nos leva a crer que tal aumento possa estar associado à abertura de bares, restaurantes e o retorno às aulas da rede privada de escolas e faculdades".

O desenvolvimento do ensino remoto no primeiro semestre de 2020 , bem como o retorno às atividades presenciais com a adoção da modalidade híbrida no segundo semestre de 2020 foram 
cercados de embates da SEDUC com professores, pais e estudantes. No primeiro momento, os questionamentos referiam-se à exclusão de parcela dos estudantes, falta de apoio aos/às professoras/es em relação a equipamentos e acesso à internet, sobrecarga de trabalho, ausência de espaços participativos para tomada de decisões sobre as estratégias pedagógicas e desconsideração dos impactos da pandemia á saúde mental dos integrantes da comunidade escolar. No segundo momento a preocupação era o risco de contaminação e a ausência de condições de trabalho presencial seguro. Vamos analisar nesse trabalho somente o primeiro momento.

\section{NARRATIVAS DE DOCENTES SOBRE AS CONDIÇÕES DE OFERTA DO ENSINO REMOTO}

A análise dos relatos das/dos docentes apontaram cinco categorias de sentido: (1) Dificuldades vivenciadas pelos/as estudantes; (2) Dificuldades vivenciadas pelas/os docentes; (3) Dificuldades vivenciadas pelas famílias; (4) Dificuldade em relação ao trabalho pedagógico; (5) Vantagens do trabalho remoto.

As/Os docentes apontaram como principais desafios enfrentados por estudantes durante a vigência do ensino remoto: indisponibilidade de equipamentos e internet, falta de espaço físico adequado para estudo, falta de apoio familiar, alterações emocionais como medo, dificuldade de concentração, agressividade ou desinteresse, dificuldades de organização de rotina de estudo. Um número significativo de estudantes saiu de Manaus durante o período mais crítico da pandemia se deslocando para áreas rurais, comunidades ribeirinhas ou municípios do interior mantendo-se inacessíveis em decorrência do isolamento de muitas comunidades amazonenses.

A adoção do ensino remoto deve levar em consideração os dados sobre o acesso a computador, tablet, internet, celular e conexão banda larga nos domicílios em Manaus/AM para não ampliar as desigualdades educacionais. A Pesquisa Nacional por Amostra de Domicílios Contínua - Tecnologia da Informação e Comunicação (Pnad Contínua TIC) de 2018, divulgada pelo Instituto Brasileiro de Geografia e Estatística (IBGE, 2019), aponta que 25,3\% da população brasileira não possui acesso à internet. Esses dados demonstram que o acesso à internet é limitado no Brasil, no entanto, para além do acesso, é importante atentar tambem para a qualidade desse acesso, pois, equipamentos tecnológicos de qualidade são de alto custo no país, impedindo que a população, em sua maioria, possua esse suporte.

A Pesquisa por Amostra Domiciliar Contínua (PNADC) de 2017 revelou que menos de $80 \%$ das residências no Brasil tem acesso à internet e na maioria dos estados, menos de $60 \%$ tem acesso a conexão por banda larga. Além disso é preciso considerar a disparidade de acesso à banda larga entre estudantes da rede privada e pública. Apenas $31 \%$ dos estudantes do ensino fundamental e $42 \%$ do ensino médio da rede pública, possuem a condição mais adequada computador/tablet e acesso com banda larga em casa. $77 \%$ e $83 \%$ dos estudantes da rede privada possuem equipamentos e banda larga, respectivamente.

Alves, Soares e Xavier (2014) ao proporem índice socioeconômico das escolas demonstram as diferenças entre estudantes de escolas privadas e públicas e a relação entre escolaridade, ocupação e renda das famílias e os resultados escolares dos/as estudantes. A maioria das matrículas (81\%) está na escola pública e apenas $19 \%$ na rede privada, entretanto, preocupa a desigualdade em relação ao nível de escolaridade dos responsáveis. No segmento público, $25 \%$ dos responsáveis têm até ensino fundamental incompleto, $24 \%$ possuem o fundamental completo, $38 \%$ ensino médio, $5 \%$ 
superior incompleto e apenas $8 \%$ têm formação em nível superior; que no segmento privado, apenas $10 \%$ não concluiu o ensino médio, $24 \%$ tem ensino médio completo, $57 \%$ são formados em nível superior e $9 \%$ não concluíram o ensino superior. A educação deve combater as desigualdades sociais e contribuir para a promoção de equidade. De acordo com Tarabini (2020) no cenário de pandemia o papel da educação pública de qualidade fica mais evidente e deve prover o oferecimento de condições mais iguais para acesso e permanência na escola.

Outro aspecto que deve ser analisado refere-se à quantidade de equipamentos disponíveis em cada residência, pois geralmente mais de um integrante da família necessita utilizá-los ao mesmo tempo. Muitas famílias têm somente um celular com pacote de dados restrito, insuficiente para a realização de atividades. $O$ acesso à internet não é universal no Brasil, não estando garantido nem aos/às educadores, nem aos/às estudantes e suas famílias, o que demanda políticas públicas que garantam a inclusão digital.

Um uso mais intenso dos recursos tecnológicos de comunicação e informação iniciou somente durante o período de isolamento social. Muitas famílias, estudantes e docentes não tinham familiaridade com essas tecnologias e ao mesmo que tempo que tinham que iniciar o seu uso, tambem estavam aprendendo como fazer. Em 2020, o Ministério da Educação divulgou uma campanha publicitária em prol da realização do Exame Nacional do Ensino Médio (ENEM) que utilizada a mensagem: "Estude, de qualquer lugar, de diferentes formas, pelos livros, internet, com a ajuda a distância dos professores" desconsiderando as condições objetivas dos/as estudantes no ano letivo e os impactos da pandemia.

O Conselho Nacional dos Direitos da Criança e do Adolescente (CONANDA) alerta que apesar de adolescentes e jovens dominarem o celular,

não possuem amplo domínio tecnológico, condições de impressão de materiais, o que pode ocasionar o abandono escolar, posterior ao período de afastamento; c) os alunos com deficiência não encontrarão a acessibilidade necessária para superar suas dificuldades (intérprete de Libras, legendagem, audiodescrição (...) crianças e alunos em situação de rua ou pertencentes à comunidades não lusófonas surdas, imigrantes, ribeirinhas, quilombolas, indígenas, do campo não têm garantido o acesso à língua portuguesa em suas famílias (CONANDA, 2020).

Em relação aos docentes, as dificuldades relatadas envolvem queixas em relação ao aumento da carga de trabalho, aumento dos gastos com energia elétrica e internet, cobranças excessivas dos gestores escolares, aumento da carga de trabalho doméstico, alterações no sono e apetite, alterações emocionais. Todas/os docentes relataram algum impacto emocional decorrente das incertezas e ameaças devido ao medo de adoecer, ao luto pela perdas de entes queridos.

Conforme expressa um docente: "A rotina sofreu uma mudança abrupta que ainda estou sentindo e passando por esse processo de adaptação que não tem sido fácil. A maior mudança sem dúvida é causada pelo distanciamento social que acarreta toda a mudança de não ter que sair de casa para exercer atividades laborais e acadêmicas nos ambientes apropriados. É um momento de aflição e cuidados maiores e isso tem entrado constantemente na rotina, em meio a tudo isso ainda fui acometido por uma gripe e venho tratando dentro de casa. Produzir academicamente em meio ao atual cenário tem sido a maior dificuldade". 
Tanto alunos/as quanto professores/as sofreram/em impactos na saúde mental em função dos estressores vivenciado nesse período de pandemia. E conforme destaca o CONANDA (2020) "este é o momento de fortalecer o diálogo democrático (...) o debate e o pensamento crítico como instrumentos pedagógicos básicos para a formação de pessoas autônomas, (...) capazes de refletir criticamente a realidade para transformá-la e torná-la mais justa". Estamos todos vivendo sob o regime da exceção e a escola deve estar a serviço da vida, transmitindo a mensagem de que, diante de uma emergência humanitária, é preciso refletir, compartilhar, construir redes de apoio ancorados no sentido da ética, que deve regular a experiência humana e o laço entre as pessoas.

Quanto às famílias, as/os docentes apontaram perceber pouca disponibilidade de tempo e/ou conhecimento para apoiar os/as filhas nas tarefas escolares. Essa pouca disponibilidade em vários casos se referia ao fato de que para aquelas pessoas não era possível ficar em casa e mesmo correndo riscos era necessário sair de casa para tentar conseguir recursos financeiros para garantir a sobrevivência da família. A pandemia representou um momento de precarização da condição econômica daqueles cuidadores que trabalhavam no mercado informal ou que ficaram desempregados. Outros familiares adoeceram, passaram por internação ou apresentaram sequelas pós COVID. As respostas no campo da educação devem considerar a complexidade e o grande desafio que é promover o direito a educação em um contexto de intensa desigualdade social. Isto é possível tomando contato com as pessoas e suas vidas.

Segundo os dados da PNAD (2018) muitos domicílios são monoparentais, sobretudo femininos, nas faixas de média baixa renda e renda baixa. É nessas faixas de renda que predominam os domicílios com casais com três filhos ou mais e as mulheres dedicam em média 18,1 horas por semana ao trabalho doméstico e aos cuidados de pessoas. Muitos pais/mães ou responsáveis que não estão desempregados, realizavam home office, o que requer o uso de computador e internet. Importante destacar a relevância da oferta de condições de conectividade. A esse respeito, estudante beneficiada pela iniciativa do UNICEF 'Rumo ao ENEM' que distribuiu Kits de conectividade para alunos do ensino médio em Manaus relatou que "compartilhava o celular com a mãe e com isso não conseguia acompanhar as aulas online e fazer pesquisas de modo eficaz, com a pandemia, fiquei desmotivada por ter dificuldade de acessar os conteúdos" (UNICEF intensifica entrega de Kits..., 2020). O apoio mostra-se decisivo para permitir o acesso.

As/Os docentes relataram dificuldades em relação ao trabalho pedagógico para a mediação pedagógica, dadas as limitações de contato com alguns estudantes, em especial, os de maior vulnerabilidade econômica bem como às demais questões apontadas acima. Aqui destaca-se a importância da oferta de meios diversificados e atentos às diferentes realidades das famílias com comunicação, informação e assistência que promova vínculos positivos e afetuosos entre responsáveis, crianças/adolescentes e docentes. As interações pautadas na escuta atenta e no diálogo e na produção de sentidos e significados sobre as experiências que vivenciam durante 0 distanciamento social pode ser uma estratégia que colabore para a elaboração das tensões vivenciadas nesse período de tantas perdas.

As estratégias de gestão educacional centralizadoras e verticais não colaboram para que docentes possam promover momentos ricos de interações livres e criativas entre crianças/adolescentes, seus pais/mães e familiares, de forma a permitir que elas/es, ainda que em isolamento, vivenciem relações de cuidado e mais ternas e solidárias em seus lares. Ao contrário disso, se os professores não têm 
espaço de escuta e participação na tomada de decisão, muito dificilmente estabelecerão com os estudantes vínculos de cuidado e diálogo.

Mesmo diante de todas as dificuldades apontadas anteriormente, as/os docentes apontam uma vantagem do trabalho remoto que é a proteção quanto ao contágio do COVID-19. E dadas as condições inadequadas de trabalho e todas as precariedades já existentes, bem como a continuidade da transmissão comunitária, este não seria o momento do retorno às atividades presenciais nas escolas. O momento deveria ser de identificação das demandas, planejamento das ações para responder a essas demandas e ampliar o apoio aos professores, estudantes e suas famílias.

O ensino remoto, pensado como uma alternativa, exige a implementação de políticas que promovam a inclusão digital da população amazônica para que não reduza a qualidade do ensino e não aprofunde as desigualdades sociais e oportunidades no universo escolar. Exige ainda o apoio aos professores para a formação, ampliar as condições de acesso a internet e a equipamentos, bem como a possibilidade de expressar suas opiniões e influenciar nas decisões.

Famílias, estudantes e docentes devem participação da construção democrática das saídas. A infraestrutura das escolas deve ser melhorada e preparada para o próximo período letivo, evitandose o aligeiramento do tempo escolar, priorizando a socialização de experiencias e conhecimentos. Deve haver espaço para o reconhecimento das singularidades e garantida a autonomia docente na construção do processo pedagógico.

\section{CONSIDERAÇÕES FINAIS}

A garantia do direito a educação deve ocorrer em conjunto com a promoção de equidade social o que pressupõe abertura ao diálogo democrático tendo como norte a garantia do direito à vida e à saúde como princípio ético. Isto será alcançado na medida em que se amplie a compreensão de que o processo pedagógico e a aprendizagem escolar é relacional.

A injustiça e a desigualdade social são características sociopolíticas marcantes no país e um dos reflexos desses fatores é a crise histórica no campo educacional. A educação brasileira enfrenta essa crise desde o Brasil-Colônia e a pandemia agravou ainda mais esse fenômeno. O ensino remoto no Amazonas, como alternativa para que a transmissão dos conteúdos educacionais continuasse foi medida importante, no entanto precisa ser aprimorado para não ser excludente e arbitrário ao desconsiderar o atual contexto socioeconômico, sanitário e em especial as demandas das pessoas envolvidas no processo.

\section{AGRADECIMENTO:}

O presente trabalho foi realizado com apoio do Programa Nacional de Cooperação Acadêmica na Amazônia - PROCAD/Amazônia da Coordenação de Aperfeiçoamento de Pessoal de Nível Superior CAPES/Brasil 


\section{REFERÊNCIAS}

ALVES, Maria Teresa Gonzaga; SOARES, José Francisco; XAVIER, Flavia Pereira. Índice socioeconômico das escolas de educação básica brasileiras. Ensaio: aval. pol. públ. Educ., Rio de Janeiro, v. 22, n. 84, p. 671-703, set. 2014. Disponível em <http://www.scielo.br/scielo.php?script=sci_arttext\&pid=S0104-

40362014000300005\&lng=pt\&nrm=iso >. acessos em 13 nov. 2020. https://doi.org/10.1590/S010440362014000300005.

ATLAS dos Objetivos de Desenvolvimento Sustentável no Amazonas. Universidade Federal do Amazonas (UFAM), Programa de Programa de Pós-Graduação em Ciências do Ambiente e Sustentabilidade na Amazônia (PPGCASA). Manaus: UFAM/PPGCASA, 2020. Disponível em: <https://www.atlasodsamazonas.ufam.edu.br/>. Acesso em: 25 set. 2020.

GARCIA-PARPET, Marie-France. Coup d'œil sur l'éducation au Brésil de Bolsonaro: Pas de financements pour la philosophie et la sociologie: il faut respecter l'argent du contribuable. Savoir/Agir, v. 49, n. 3, p. 95-103, 2019.

CONSELHO ESTADUAL DE EDUCAÇÃO DO AMAZONAS (CEE/AM). Resolução No 30 de 18 de março de 2020. Dispõe sobre o regime especial de aulas não presenciais no Sistema de Ensino do Estado do Amazonas, como medida preventiva à disseminação do COVID-19. Disponível em http://www.educacao.am.gov.br/wp-content/uploads/2020/03/Resolucao-30-ano-2020-1.pdf Acesso 20 de setembro de 2020.

CONSELHO NACIONAL DOS DIREITOS DA CRIANÇA E DO ADOLESCENTE. Manifestação do CONANDA sobre o direito à educação de crianças e adolescentes durante a pandemia do covid-19. Brasília, DF: CONANDA, 2000.

FUNDAÇÃO DE VIGILÂNCIA SANITÁRIA (FVS-AM). Boletim diário de casos COVID-19. Disponível em http://www.fvs.am.gov.br/media/publicacao/13_12_20_BOLETIM_DI\%C3\%81RIO_DE_CASOS_COV ID-19.pdf Acesso em 10 de dezembro de 2020

INSTITUTO BRASILEIRO DE GEOGRAFIA E ESTATÍSTICA (IBGE). Pesquisa por Amostra Domiciliar Contínua (PNADC) de 2017. Brasília, DF: IBGE, 2017.

INSTITUTO BRASILEIRO DE GEOGRAFIA E ESTATÍSTICA (IBGE). Pesquisa Nacional por Amostra de Domicílios Contínua - Tecnologia da Informação e Comunicação (Pnad Contínua TIC) de 2018. Brasília, DF: IBGE, 2019.

SECRETARIA DE ESTADO DE EDUCAÇÃO E DESPORTO DO AMAZONAS (SEDUC). Gabinete do Secretário. Portaria GS No 311 de 20 de março de 2020. Institui, no âmbito da rede pública estadual de ensino do Amazonas, o regime especial de aulas não presenciais, para a educação básica, como medida preventiva à disseminação do COVID-19. Disponível em http://www.educacao.am.gov.br/wp-content/uploads/2020/03/Portaria-GS-311-de-20-03-20-20-032020-5-26-PM-1.pdf Acesso 20 de setembro de 2020. 2020a.

SECRETARIA DE ESTADO DE EDUCAÇÃO E DESPORTO DO AMAZONAS (SEDUC). Secretaria Executiva Adjunta Pedagógica. Departamento de Políticas e Programas Educacionais. Diretrizes Pedagógicas para o Regime Especial de Aulas Não Presenciais. Disponível em http://www.educacao.am.gov.br/wp-content/uploads/2020/03/DIRETRIZES-PEDAGoGICAS23.03.pdf Acesso em 20 de setembro de 2020. 2020b

SECRETARIA DE ESTADO DE EDUCAÇÃO E DESPORTO DO AMAZONAS (SEDUC). Secretaria Executiva Adjunta Pedagógica. Nota de Esclarecimento à Comunidade Escola Acerca do Regime Especial de Aulas Não Presenciais. Disponível em http://www.educacao.am.gov.br/wp- 
content/uploads/2020/03/NOTA-DE-ESCLARECIMENTO-A-COMUNIDADE-ESCOLAR.pdf Acesso 20 de setembro de 2020. 2020c.

MICHETTI, Miqueli. Entre a legitimação e a crítica: as disputas acerca da Base Nacional Comum Curricular. Revista Brasileira de Ciências Sociais. v. 35, n.102, p. 1-19, 2020.

TARABINI, Aina. ¿Para qué sirve la escuela? Reflexiones sociológicas en tiempos de pandemia global. RASE: revista de sociología de la educación. v. 13, n. 2, p. 145-155, 2020.

UNICEF intensifica entrega de Kits 'Rumo ao Enem', em Manaus. Portal A Crítica. Caderno Ação Social. 10/12/2020. Disponível em https://www.acritica.com/channels/educacao/news/unicefintensifica-entrega-de-kits-rumo-ao-enem-em-manaus. Acesso em 10 de dezembro de 2020. 